\title{
Metabolic correction therapy for the treatment of a diabetic foot ulcer: A case report
}

\author{
Jomaris Centeno ${ }^{1}$, Marilís Navarrete ${ }^{1}$, Miguel J Berdiel ${ }^{2}$ and Michael Gonzalez ${ }^{3 *}$ \\ ${ }^{1}$ San Juan Bautista School of Medicine, Caguas, Puerto Rico \\ ${ }^{2}$ Berdiel Clinic, Ponce, Puerto Rico \\ ${ }^{3}$ University of Puerto Rico, Medical Sciences Campus, School of Public Health, San Juan, Puerto Rico
}

\begin{abstract}
Diabetes is a chronic metabolic disease that more than 29 million Americans suffer. Approximately, 1.4 million new cases are reported every year with more than 200 thousand deaths listing diabetes as the cause of death. Uncontrolled levels of glucose, known as hyperglycemic state, can eventually lead to complications such as retinopathy, hypertension, neuropathy, foot ulcers and/or amputations. Foot ulcers develop in $15 \%$ of diabetic patients and increase the risk of lower-limb amputations. This is a case report of a 63 year old patient with Type 1 diabetes mellitus presented to the clinic because of a foot ulcer after seven previous medical recommendations of amputation of the limb. The patient was started on a Metabolic Correction Therapy and GlucoCoR for glucose blood level regulation. Upon six months the foot ulcer healed and foot sensation was recovered. We propose this therapy as an affordable effective treatment mechanism to lower the risk of developing diabetic complications, having great beneficial effect in the quality of life and survival rate of diabetic patients.
\end{abstract}

\section{Introduction}

Diabetes is a chronic metabolic disease which, according to the World Health Organization, affects more than 400 million people [1]. In 2012, more than 29 million Americans suffered from diabetes and around 1.4 million new cases of diabetes are reported each year. ${ }^{2}$ In 2010,234,051 death certificates indicated diabetes as the cause of death and 69,071 indicated diabetes as the underlying cause of death, placing this disease as the seventh cause of death in the United States [2]. It is believed that these numbers are underestimated and data of deaths with diabetes as underlying cause of death should be higher.

Diabetes is characterized by higher than normal blood glucose level as a result of either an absolute (Type 1) or relative (Type 2) deficiency of insulin or insulin resistance. When uncontrolled, it can eventually lead to complications such as retinopathy, hypertension, neuropathy, foot ulcers and/or amputations [3]. Interestingly, the hyperglycemic state in diabetes mellitus has been related with an increased production of reactive oxygen species (ROS) and a decrease in endogenous oxidoreductant molecules, this imbalance leads to oxidative stress [4]. Subsequently, oxidative stress is considered to have a big role in nerve damage (neurodegeneration) and is most prominent in longer sensory axons by way of mitochondrial dysfunction $[5,6]$.

For 2009-2012, it was reported that $71 \%$ of diabetics older than 18 years had hypertension or used of medication to lower blood pressure [2]. Neuropathy, the progressive loss of somatic and autonomic nerve fibers [5] is present from mild to severe form in $60-70 \%$ of diabetic patients [2]. Nerve damage in the feet of diabetic patients increases the chance of damage to the limbs that result in foot ulcers [1].

Diabetic foot ulcers are slow or non-healing lesions that form on feet of diabetic patients. They develop in around $15 \%$ of diabetic patients [7] and increase the risk of lower-limb amputation [1,8]. This are 10 to 20 times higher among people with diabetes and is one of the most expensive complications of this disease [1]. 60\% of nontraumatic lower-limb amputations are performed in people with diabetes. As reported in 2010, around 73,000 nontraumatic lower-limb amputations are performed in adults with diabetes annually [2].

Although amputation is advised when infection threatens the overall health of the patient, this course of action has a negative effect on mobility and lifestyle of the patients. Diabetics with these complications have a poor quality of life, and are more likely to have a higher risk of premature death [1].

In the search of an affordable effective treatment mechanism that lowers the risk of developing diabetic complications, this case reports the adoption of an unconventional treatment based on a metabolic correction therapy in the case of a diabetic foot ulcer, resulting in complete healing of the wound and regaining neural sensation and saving of the affected limb.

\section{Case report}

A 63 year old patient with Type 1 diabetes mellitus presented to the clinic because of a foot ulcer. The ulcer was located on the posterior surface (sole) of the right foot at the level of the first phalange extending to the level of the first metatarsal (Figure 1a) and to the medial aspect of the foot (Figure 1b). The ulcer presented necrotic tissue and loss of sensation, even to painful stimulus. The patient came to the clinic after seven previous medical recommendations of amputation of the limb.

Correspondence to: Michael Gonzalez, University of Puerto Rico, Medical Sciences Campus, School of Public Health, San Juan, Puerto Rico, E-mail: michael.gonzalez5@upr.edu

Received: September 02, 2017; Accepted: September 20, 2017; Published: September 22, 2017 

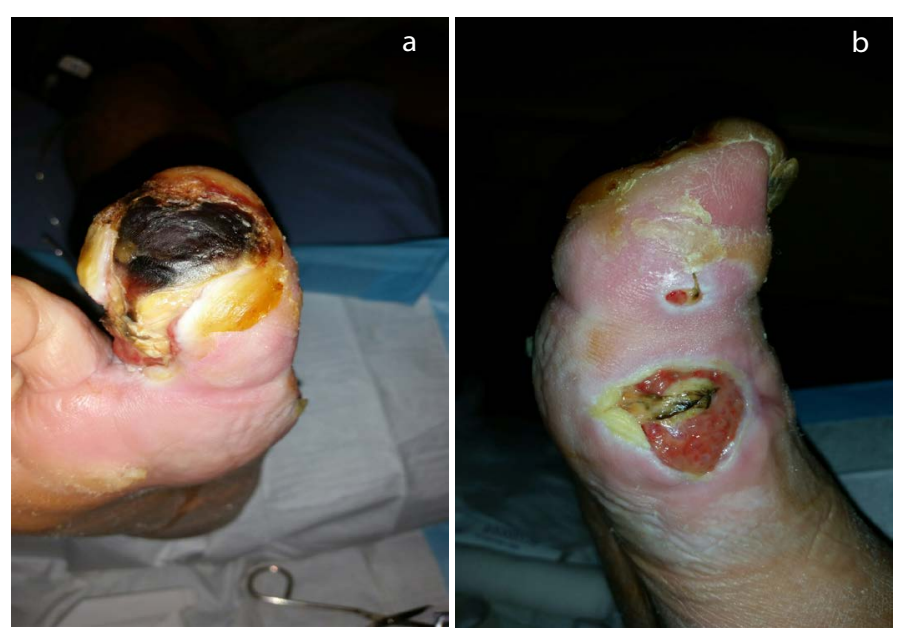

Figure 1. Posterior aspect (a) and medial aspect (b) of the patient's foot ulcer at the beginning of treatment.

Previous medication for blood glucose level control was discontinued (Metformin $1000 \mathrm{mg} 2$ bid and Glucotrol XL $10 \mathrm{mg}$ ) except for Humulin 70/30 10 units/mL subcutaneous tid after eating. 2 GlucoCoR ${ }^{\star}$ pills in the morning and 2 in the afternoon, and Lantus 40 units $/ \mathrm{mL}$ subcutaneous in the afternoon where added as glucose control medication.

A metabolic correction treatment was started. It consisted of a strict Paleolithic Diet of low carbohydrate intake of organic vegetables and nuts combined with grass-fed meat, free-range poultry and wild-caught fish. Processed food and refined sugar products were eliminated from the diet. This diet was supplemented with the following multivitamins and minerals: CoQ10 $100 \mathrm{mg}$ tid, R-alpha lipoic acid $300 \mathrm{mg}$ bid, Acetyl L Carnitine 500 mg bid, Magnesium Citrate 500 mg bid, Omega-3's 1 g tid, Mixed phospholipids $100 \mathrm{mg}$ qd, Vitamin D3 10,000 IU qd, B complex $1 \mathrm{cc}$ sublingual tid.

In addition, the patient was administered high doses of intravenous ascorbic acid (IVAA) three times a week. The protocol was started with a weekly increase in the dosage of the IVAA infusion, until reaching the optimal dosage that was continued (75 gIVAA). First week $15 \mathrm{~g}$ three times a week, second week $25 \mathrm{~g}$ three times a week, third week $50 \mathrm{~g}$ three times a week, and fourth week $75 \mathrm{~g}$ three times a week. The patient also had hyperbaric oxygen therapy (HBOT) two times a week at $1.5 \mathrm{~atm}$ for 45 minutes.

On the second visit, minimal granulation tissue was observed and treatment was continued. As the patient continued treatment the ulcer improved remarkably (Figures 2 and 3 ) and was able to feel sensation on the foot and walk comfortably.

\section{Discussion}

Metabolic correction therapy consists of supplementing the body with micronutrients (vitamins and minerals) that are normal in the body and that are required for normal health [9]. It is based on the fact that the Standard American Diet is leading to nutritional insufficiencies and deficiencies [10]. This diet consists of high calorie processed foods and refined sugars. These types of food do not meet the recommended nutritional requirements [9]. The lack of nutrients impairs progression of biochemical reactions in cells by hindering enzymatic function and by prioritizing the available nutrients to the most life preserving reactions which can lead to cell damage [9].
Adopting a Paleolithic diet helps improve patient's health in more than one aspect. It decreases the amount of sugar intake for a better management of the disease and for lowering the risk of developing hyperglycemic induced complications. At the same time, it increases the amount of dietary nutrients and vitamins by eating nutrient rich quality products. This diet, in combination with supplementation of multivitamins and vitamins, aid the body by replenishing micronutrients and cofactors necessary for normal biologic function [9]. Metabolic correction is considered to be reached when the body reaches optimal levels of nutrients. At this stage the cells and enzymes have enough to correct biochemical disruptions previously impaired by nutrient deficiency or insufficiencies [9]. At a biochemical level, this homeostatic state induces an overall metabolic and physiologic improvement in which the body has enough nutrients and energy to heal itself [11].

GlucoCoR ${ }^{\oplus}$ was incorporated to the medication for blood glucose level control. As part of this therapy, it acts as a metabolic corrector formulated to maintain normal blood glucose levels, decrease homocysteine levels, protect against oxidative damage and promote healthy tissues and collagen formation.

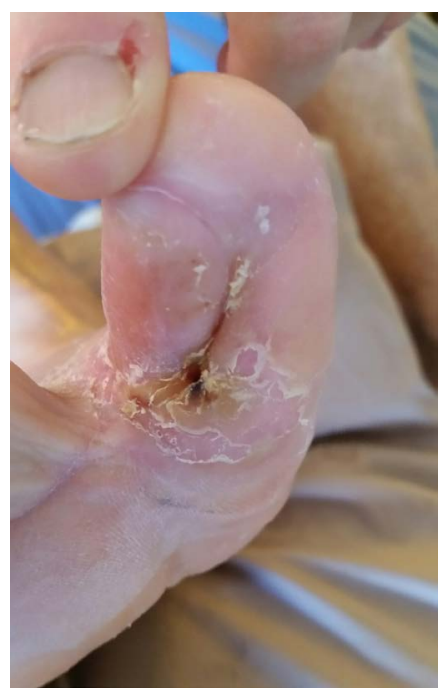

Figure 2. Foot ulcer at month 4 of treatment.

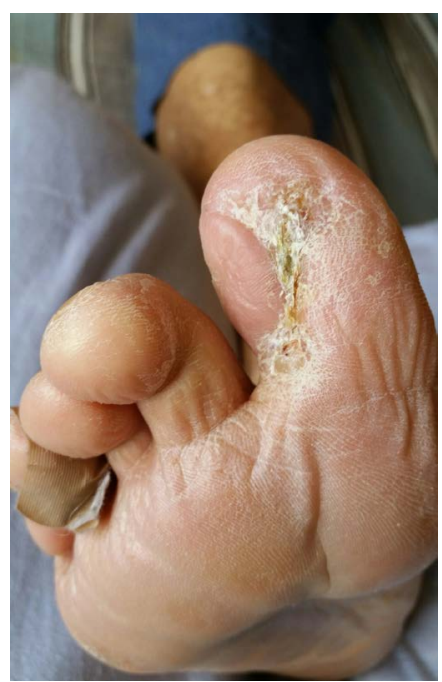

Figure 3. Foot ulcer at month 6 of treatment. 
Vitamin C can enter cells through glucose transporters [12], it can be taken into the cell in the place of glucose, decreasing its intracellular levels and, thus, helping lower glucose induced damage. Vitamin C can also enter the mitochondria and protect the membrane from ROS [13] and improve mitochondrial energy metabolism [14]. It could also have an ergogenic capacity by donating electrons to the electron transport chain resulting in increased energy production [12]. It is also essential for synthesis and maintenance of collagen, necessary for connective tissue formation [12]. Consequently, Vitamin C should be considered as a treatment for slow healing foot ulcers $[12,15]$.

HBOT increases the oxygen that is delivered to tissues. Given that diabetic foot ulcers are associated with ischemia, this therapy has been proposed to increase wound healing [16]. Studies have demonstrated that HBOT is a promising adjunctive treatment because it has been reported to enhance foot ulcer healing and reduce limb amputation in diabetic patients $[17,18]$.

The combination of conventional medicine to control blood glucose levels with the metabolic correction therapy is an excellent option for diabetic patients, as it will help lower the risk of developing complications or reduce the effects of existing ones. Considering the high cost of diabetes and its complications, this therapy is an inexpensive option with low toxicity [12]. As with the patient, metabolic correction therapy could reduce the need of additional drugs, treatments and/ or surgeries, reducing expenses and possible side effects. This therapy would also have great beneficial effect in the quality of life and survival rate of diabetic patients.

\section{References}

1. World Health Organization (2016) Global Report on Diabetes.

2. American Diabetes Association (2017) Statistics About Diabetes; http://www.diabetes. org/diabetes-basics/statistics/.

3. Ullah F, Afridi AK, Rahim F, Ashfaq M, Khan S, et al. (2015) Knowledge Of Diabetic Complications In Patients With Diabetes Mellitus. J Ayub Med Coll Abbottabad 27: 360-363. [Crossref]

4. Bonnefont-Rousselot D (2004) The Role of Antioxidant Micronutrients in the Prevention of Diabetic Complications. Treat Endocrinol 3: 41-52. [Crossref]
5. Tecilazich F, Dinh TL, Veves A (2012) Role of Peripheral Neuropathy in the Development of Foot Ulceration and Impaired Wound Healing in Diabetes Mellitus. In: Nutritional and Therapeutic Interventions for Diabetes and Metabolic Syndrome. Elsevier; 185-194.

6. Hernández-Beltrán N, Moreno CB, Gutiérrez-Álvarez ÁM (2013) Contribution of mitochondria to pain in diabetic neuropathy. Endocrinol Nutr 60: 25-32. [Crossref]

7. Mayfield JA, Reiber GE, Sanders LJ, Janisse D, Pogach LM (1998) Preventive foot care in people with diabetes. Diabetes Care 21: 2161-2177. [Crossref]

8. Futrega K, King M, Lott WB, Doran MR (2014) Treating the whole not the hole: necessary coupling of technologies for diabetic foot ulcer treatment. Trends Mol Med 20: 137-142. [Crossref]

9. Gonzalez MJ, Miranda-Massari JR (2016) Metabolic Correction: A Functional Biochemical Therapeutic Approach. Townsend Letter.

10. Gonzalez MJ, Miranda-Massari JR (2013) Orthomolecular Medicine: The Best Cost Effective, Rational and Scientific Choice for Disease Treatment. Journal of Orthomolecular Medicine 28: 5-8.

11. González MJ, Miranda-Massari JR, Mora EM, Cruzado NA, Jiménez I, et al. (2000) Integrative medicine: a paradigm shift in medical education and practice. $P R$ Health Sci J 19: 389-392. [Crossref]

12. Gonzalez MJ (2005) Orthomolecular Oncology Review: Ascorbic Acid and Cancer 25 Years Later. Integr Cancer Ther 4: 32-44. [Crossref]

13. Sagun K, Cárcamo JM., Golde DW (2005) Vitamin C enters mitochondria via facilitative glucose transporter 1 (Glut1) and confers mitochondrial protection against oxidative injury. FASEB J 19: 1657-1667. [Crossref]

14. Luo G, Xie ZZ, Liu FY, Zhang GB (1998) Effects of vitamin C on myocardial mitochondrial function and ATP content in hypoxic rats. Zhongguo Yao Li Xue Bao 19: 351-355. [Crossref]

15. Christie-David DJ, Gunton JE (2017) Vitamin C deficiency and diabetes mellitus easily missed? Diabet Med 34: 294-296. [Crossref]

16. Kranke P, Bennett MH, Martyn-St James M, Schnabel A, Debus SE, et al. (2015) Hyperbaric oxygen therapy for chronic wounds. In: Kranke P (Ed.), Cochrane Database of Systematic Reviews. Vol 11. Chichester, UK: John Wiley \& Sons, Ltd; 28-34.

17. Londahl M, Katzman P, Nilsson A, Hammarlund C (2010) Hyperbaric Oxygen Therapy Facilitates Healing of Chronic Foot Ulcers in Patients With Diabetes. Diabetes Care 33: 998-1003. [Crossref]

18. Bishop AJ, Mudge E (2014) Diabetic foot ulcers treated with hyperbaric oxygen therapy: A review of the literature. Int Wound J 11: 28-34. [Crossref]

Copyright: $(02017$ Centeno J. This is an open-access article distributed under the terms of the Creative Commons Attribution License, which permits unrestricted use, distribution, and reproduction in any medium, provided the original author and source are credited. 